\title{
Tandem Mass Spectrometry for Structural Identification of Sesquiterpene Alkaloids from the Stems of Dendrobium nobile Using LC-QToF*
}

Authors

Affiliations
Yan-Hong Wang ${ }^{1}$, Bharathi Avula ${ }^{1}$, Naohito Abe ${ }^{1}$, Feng Wei ${ }^{2}$, Mei Wang ${ }^{1}$, Shuang-Cheng Ma ${ }^{2}$, Zulfiqar Ali ${ }^{1}$, Mahmoud A. Elsohly ${ }^{1,3}$, Ikhlas A. Khan ${ }^{1,4,5}$

The affiliations are listed at the end of the article

\author{
Key words \\ - Dendrobium nobile \\ - Orchidaceae \\ - shi hu \\ - dendrobine \\ - nobilonine \\ - UHPLC-QToF/MS \\ - tandem mass \\ - sesquiterpene alkaloids
}

received October 20,2014 revised January 19, 2016 accepted February 4, 2016

\section{Bibliography}

DOI http://dx.doi.org/

$10.1055 / \mathrm{s}-0042-103031$

Published online April 7, 2016

Planta Med 2016; 82: 662-670

(c) Georg Thieme Verlag KG

Stuttgart · New York .

ISSN 0032-0943

\section{Correspondence}

\section{Ikhlas A. Khan}

National Center for Natural

Products Research

University of Mississippi

University, MS 38677

United States

Phone: + 16629157821

Fax: +16629157989

ikhan@olemiss.edu

\begin{abstract}
$\nabla$

Dendrobium nobile is one of the fundamental herbs in traditional Chinese medicine. Sesquiterpene alkaloids are the main active components in this plant. Due to weak ultraviolet absorption and low content in $D$. nobile, these sesquiterpene alkaloids have not been extensively studied using chromatographic methods. Herein, tandem mass spectrometry combined with liquid chromatography separation provides a tool for the identification and characterization of the alkaloids from D. nobile. A total of nine sesquiterpene alkaloids were characterized by ultrahigh-performance liquid chromatography tandem mass spectrometry. These alkaloids can be classified into two subgroups that are represented by dendrobine and nobilonine. Tandem mass spectrometric studies
\end{abstract}

\section{Introduction}

Dendrobium nobile Lindl. (Orchidaceae) is also known as Noble Dendrobium or jin-chai-shi-hu. The stems of $D$. nobile are one of the fundamental herbs in traditional Chinese medicine (TCM) used for the treatment of chronic gastritis to lower cholesterol levels, regulate blood pressure, promote the secretion of saliva, reduce fever, and strengthen the immune system [1]. Phytochemical investigations revealed alkaloids, aromatic compounds, sesquiterpenoids, and polysaccharides in $D$. nobile [2]. The polysaccharides, alkaloids, and aromatics were reported as the main components responsible for the biological activ-

* Part of this work has been presented as a poster presentation at the American Society of Pharmacognosy 2014 Annual Meeting - Held in conjunction with the 14th Annual Oxford International Conference on the Science of Botanicals. The abstract of the poster was published in Planta Medica 2014; 80: PD110; DOI: 10.1055/s-00341382531 revealed the fragmentation pathways of these two subgroup alkaloids that were used for the identification and characterization of other alkaloids in D. nobile. Characterization of these alkaloids using accurate mass and diagnostic fragments provided a reliable methodology for the analysis of $D$. nobile by ultrahigh-performance liquid chromatography tandem mass spectrometry. The limit of detection was defined as the signalto-noise ratio equal to $3: 1$. Limits of detection of dendrobine and nobilonine were less than $30 \mathrm{ng} /$ $\mathrm{mL}$. The developed method was applied for the analysis of various Dendrobium species and related dietary supplements. Alkaloids were identified from $D$. nobile, but not detected from commercial samples including 13 other Dendrobium species and the 7 dietary supplements. 


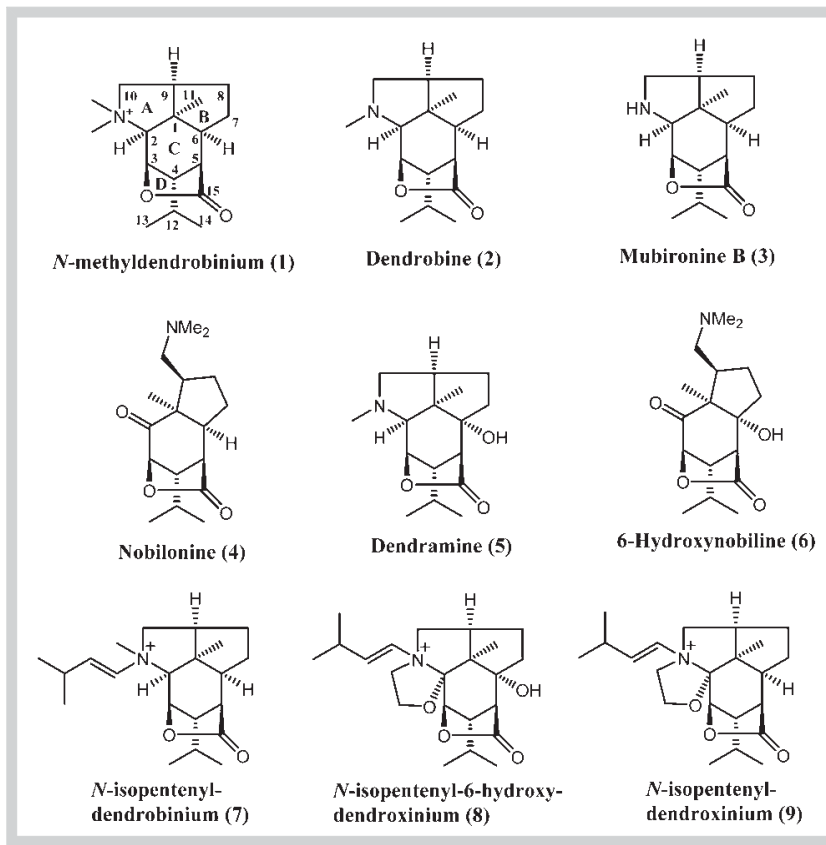

Fig. 1 Structures of sesquiterpene alkaloids: $1 \mathrm{~N}$-methyldendrobinium, 2 dendrobine, $\mathbf{3}$ mubironine B, $\mathbf{4}$ nobilonine, 5 dendramine, $\mathbf{6}$ 6-hydroxynobiline, $7 \mathrm{~N}$-isopentenyldendrobinium, $8 \mathrm{~N}$-isopentenyl-6-hydroxydendroxinium, and $\mathbf{9} \mathrm{N}$-isopentenyldendroxinium.

12], capillary electrophoresis (CE) [13], and gas chromatography (GC) [14]. In two LC-UV methods, one or two alkaloids were used in the analysis of Dendrobium species $[9,10]$. UV detection is considered inappropriate for dendrobine-type alkaloids because these compounds have extremely weak ultraviolet absorption due to the absence of chromophore in the core skeleton. Mass spectrometry detection, especially high-resolution mass spectrometric techniques such as quadrupole time-of-flight (QToF), is a useful tool for the identification of sesquiterpene alkaloids since it can provide accurate mass and structural information.

As a part of a USFDA research program for the science based authentication of dietary supplements, an ultrahigh-performance liquid chromatograph (UHPLC) coupled with an electrospray ionization quadrupole time-of-flight mass spectrometry (UHPLCESI/QToF MS) method was developed. The UHPLC coupled with tandem mass spectrometric method was designed to identify and structurally characterize sesquiterpene alkaloids from Dendrobium species and dietary supplements claiming to contain D. nobile.

\section{Results and Discussion}

$\nabla$

In a preliminary test for the characterization of dendrobine-type alkaloids, different columns were applied for the analysis of D. nobile extracts (NCNPR \#13021). The columns tested were Acquity UPLC BEH Shield RP18 $(100 \times 2.1 \mathrm{~mm}$ i.d., $1.7 \mu \mathrm{m})$, UPLC BEH C18 $(100 \times 2.1 \mathrm{~mm}$ i.d., $1.7 \mu \mathrm{m})$, and UPLC HSS T3 $(100 \times 2.1 \mathrm{~mm}$ i.d., $1.8 \mu \mathrm{m})$. The best separation and peak shape were achieved on the UPLC BEH Shield RP18 column. Different solvent systems such as acetonitrile/water and methanol/water were investigated to determine the best separation conditions for the sesquiterpene alkaloids. Optimal chromatographic sepa- ration was observed by using acetonitrile with $0.05 \%$ formic acid $(\mathrm{v} / \mathrm{v})$ and $0.05 \%$ formic acid in water as the mobile phase. Authentic plant sample \#13021 was extracted with 100\% methanol and $70 \%$ methanol (methanol/water $=70: 30, \mathrm{v} / \mathrm{v}$ ), respectively. The peak areas of the major alkaloids (compounds 2, 4, 5, and 7-9) did not differ significantly. However, considering the higher solubility of alkaloids' salts in aqueous solution, $70 \%$ methanol was used as the extraction solvent.

Dendrobine-type sesquiterpene alkaloids from $D$. nobile have a basic skeleton that consists of one picrotoxan-type sesquiterpenoid combined with a five-membered C2-C9-linked N-heterocycle and a C3-C5-linked lactone ring ( $\bullet$ Fig. 1 ).

The characterization of the sesquiterpene alkaloids in D. nobile authentic plant sample \#13021 was based on two approaches: (1) unambiguous identification by comparing with reference standards (dendrobine and nobilonine) in terms of the retention time, accurate mass, and MS/MS fragment ions, and (2) tentative characterization of compounds without reference by analyzing accurate mass and MS/MS product ions, comparing key fragments (diagnostic fragments) with the fragmentation pattern of dendrobine and nobilonine, and surveying the literature. As a result, a total of nine alkaloids were identified or tentatively characterized. Structural information including the retention time, precursor ions $\left([\mathrm{M}]^{+} /[\mathrm{M}+\mathrm{H}]^{+}\right)$, MS/MS product ions, and the composition of fragments for these compounds is summarized in $\triangle$ Table 1. The base peak ion (BPI) chromatogram of the extracts of D. nobile and extracted ion chromatograms (EIC) of the individual alkaloids are shown in $\boldsymbol{0}$ Fig. 2 . The MS/MS spectra of nine alkaloids are presented in $\bullet$ Fig. $\mathbf{3 .}$

In the developed UHPLC-MS method, the standard compound dendrobine (compound $\mathbf{2}$ in $\mathbf{O}$ Fig. $\mathbf{2}$ ) produced a protonated molecular ion at $\mathrm{m} / z 264.1956\left([\mathrm{M}+\mathrm{H}]^{+}, \mathrm{C}_{16} \mathrm{H}_{26} \mathrm{NO}_{2}{ }^{+}\right.$, calcd. 264.1958). The limit of detection (LOD) was defined as the signal-to-noise ratio equal to $3: 1$. The LOD of dendrobine was less than $30 \mathrm{ng} / \mathrm{mL}$. Collision-induced dissociation (CID) of the [M + $\mathrm{H}]^{+}$ions led to the cleavage of the lactone ring between $\mathrm{C} 3$ and C5. After the neutral losses of $\mathrm{H}_{2} \mathrm{O}$ from C-3 and $\mathrm{CO}$ from C-5, product ions at $m / z 218.1901\left(\mathrm{C}_{15} \mathrm{H}_{24} \mathrm{~N}^{+}\right)$were generated. The successive cleavages of $\mathrm{C} 4-\mathrm{C} 12$ and $\mathrm{C} 1-\mathrm{C} 11$ generated ions at $\mathrm{m} / z 176.1433\left[218-\mathrm{C}_{3} \mathrm{H}_{6}\right]^{+}$and $160.1111\left[176-\mathrm{CH}_{4}\right]^{+}$, respectively. The fragment $\left[176-\mathrm{CH}_{4}\right]^{+}$consecutively yielded ions at $\mathrm{m} / \mathrm{z}$ 145.0878 and 133.1003 corresponding to fragments $\left[160-\mathrm{CH}_{3}\right]^{+}$ and $[160-\mathrm{HCN}]^{+}$, respectively. Product ions at $\mathrm{m} / z 119.0848$ and 105.0690 came from fragment $[160-\mathrm{HCN}]^{+}$corresponding to the losses of $\mathrm{CH}_{2}$ and $\mathrm{C}_{2} \mathrm{H}_{4}$, respectively. The proposed fragmentation pathway of dendrobine is shown in $\odot$ Fig. 4. The neutral losses of 28,18 , and $42 \mathrm{Da}$ corresponding to the $\mathrm{CO}, \mathrm{H}_{2} \mathrm{O}$, and $\mathrm{C}_{3} \mathrm{H}_{6}$ moieties, respectively, are diagnostic for the characterization of the core skeleton of dendrobine-type alkaloids, which are also observed in the MS/MS spectra of compounds 2, 3, 5, and 7-9 in - Table 1.

From the methanolic extract of $D$. nobile, compound 2 was unambiguously identified as dendrobine. Compound 3 produced ions at $\mathrm{m} / \mathrm{z} 250.1802\left([\mathrm{M}+\mathrm{H}]^{+}, \mathrm{C}_{15} \mathrm{H}_{24} \mathrm{NO}_{2}{ }^{+}\right.$, calcd. 250.1802), and the characteristic fragment ions at $\mathrm{m} / \mathrm{z} 232.1702,204.1756$, and 190.1229 were found in accordance with the fragmentation pattern of dendrobine-type alkaloids. According to the molecular formula of the $[\mathrm{M}+\mathrm{H}]^{+}$ion, compound $\mathbf{3}$ was 14 Da less than that of dendrobine 2, indicating a loss of a methylene moiety in $\mathbf{2}$. Based on the aforementioned information, compound 3 could be tentatively identified as mubironine $\mathrm{B}$, which has been reported 
Table 1 Positive ESI/QToF/MS/MS data of alkaloid compounds identified from D. nobile: $\mathbf{1}$ N-methyldendrobinium, 2 dendrobine, 3 mubironine B, 4 nobilonine, 5 dendramine, 6 6-hydroxynobiline, $7 \mathrm{~N}$-isopentenyldendrobinium, $8 \mathrm{~N}$-isopentenyl-6-hydroxydendroxinium, and $\mathbf{9} \mathrm{N}$-isopentenyldendroxinium.

\begin{tabular}{|c|c|c|c|c|c|c|c|c|}
\hline Peak & $\begin{array}{l}t_{R} \\
(\min )\end{array}$ & $\begin{array}{l}\text { CE } \\
\text { (ev) }\end{array}$ & $\begin{array}{l}\text { Precursor lon } \\
(\mathrm{m} / \mathrm{z})\end{array}$ & $\begin{array}{l}\text { Experimental } \\
\text { MS/MS (m/z) }\end{array}$ & $\begin{array}{l}\text { Calculated } \\
\text { MS/MS (m/z) }\end{array}$ & $\begin{array}{l}\text { Elemental } \\
\text { composition }\end{array}$ & $\begin{array}{l}\text { Error } \\
\text { (ppm) }\end{array}$ & $\begin{array}{l}\text { Structural } \\
\text { elucidation }\end{array}$ \\
\hline \multirow[t]{5}{*}{1} & \multirow[t]{5}{*}{3.50} & \multirow[t]{5}{*}{40} & \multirow[t]{5}{*}{278.2120} & 278.2120 & 278.2115 & $\mathrm{C}_{17} \mathrm{H}_{28} \mathrm{NO}_{2}{ }^{+}$ & 3.10 & \multirow{5}{*}{$\begin{array}{l}\mathrm{N} \text {-methylden- } \\
\text { drobinium }\end{array}$} \\
\hline & & & & 190.1621 & 190.1596 & $\mathrm{C}_{13} \mathrm{H}_{20} \mathrm{~N}^{+}$ & 13.14 & \\
\hline & & & & 145.0878 & 145.0886 & $\mathrm{C}_{10} \mathrm{H}_{11} \mathrm{~N}^{+}$. & -6.01 & \\
\hline & & & & 119.0846 & 119.0855 & $\mathrm{C}_{9} \mathrm{H}_{11}^{+}$ & -8.41 & \\
\hline & & & & 105.0692 & 105.0699 & $\mathrm{C}_{8} \mathrm{H}_{9}{ }^{+}$ & -2.50 & \\
\hline \multirow[t]{10}{*}{2} & \multirow[t]{10}{*}{4.12} & \multirow[t]{10}{*}{40} & \multirow[t]{10}{*}{264.1956} & 264.1956 & 264.1958 & $\mathrm{C}_{16} \mathrm{H}_{26} \mathrm{NO}_{2}^{+}$ & -0.76 & \multirow[t]{10}{*}{ dendrobine } \\
\hline & & & & 246.1865 & 246.1852 & $\mathrm{C}_{16} \mathrm{H}_{24} \mathrm{NO}^{+}$ & 4.96 & \\
\hline & & & & 236.2000 & 236.2009 & $\mathrm{C}_{15} \mathrm{H}_{26} \mathrm{NO}^{+}$ & -3.83 & \\
\hline & & & & 218.1901 & 218.1903 & $\mathrm{C}_{15} \mathrm{H}_{24} \mathrm{~N}^{+}$ & -0.91 & \\
\hline & & & & 176.1433 & 176.1434 & $\mathrm{C}_{12} \mathrm{H}_{18} \mathrm{~N}^{+}$ & -0.48 & \\
\hline & & & & 160.1111 & 160.1121 & $\mathrm{C}_{11} \mathrm{H}_{14} \mathrm{~N}^{+}$ & -5.20 & \\
\hline & & & & 145.0878 & 145.0886 & $\mathrm{C}_{10} \mathrm{H}_{11} \mathrm{~N}^{+}$. & -4.49 & \\
\hline & & & & 133.1003 & 133.1012 & $\mathrm{C}_{10} \mathrm{H}_{13}{ }^{+}$ & -5.48 & \\
\hline & & & & 119.0848 & 119.0855 & $\mathrm{C}_{9} \mathrm{H}_{11}^{+}$ & -2.26 & \\
\hline & & & & 105.0690 & 105.0699 & $\mathrm{C}_{8} \mathrm{H}_{9}^{+}$ & -3.08 & \\
\hline \multirow[t]{10}{*}{3} & \multirow[t]{10}{*}{4.38} & \multirow[t]{10}{*}{30} & \multirow[t]{10}{*}{250.1802} & 250.1802 & 250.1802 & $\mathrm{C}_{15} \mathrm{H}_{24} \mathrm{NO}_{2}^{+}$ & 0.00 & mubironine B \\
\hline & & & & 232.1702 & 232.1696 & $\mathrm{C}_{15} \mathrm{H}_{22} \mathrm{NO}^{+}$ & 2.71 & \\
\hline & & & & 204.1756 & 204.1747 & $\mathrm{C}_{14} \mathrm{H}_{22} \mathrm{~N}^{+}$ & 4.43 & \\
\hline & & & & 190.1229 & 190.1226 & $\mathrm{C}_{12} \mathrm{H}_{16} \mathrm{NO}^{+}$ & 1.58 & \\
\hline & & & & 175.0989 & 175.0992 & $\mathrm{C}_{11} \mathrm{H}_{13} \mathrm{NO}^{+}$ & -1.71 & \\
\hline & & & & 162.1263 & 162.1277 & $\mathrm{C}_{11} \mathrm{H}_{16} \mathrm{~N}^{+}$ & -8.64 & \\
\hline & & & & 145.0882 & 145.0886 & $\mathrm{C}_{10} \mathrm{H}_{11} \mathrm{~N}^{+}$. & -2.28 & \\
\hline & & & & 133.1016 & 133.1012 & $\mathrm{C}_{10} \mathrm{H}_{13}^{+}$ & 2.48 & \\
\hline & & & & 119.0845 & 119.0855 & $\mathrm{C}_{9} \mathrm{H}_{11}^{+}$ & -7.51 & \\
\hline & & & & 105.0699 & 105.0699 & $\mathrm{C}_{8} \mathrm{H}_{9}{ }^{+}$ & 0.00 & \\
\hline 4 & 5.17 & 30 & 294.2067 & 294.2067 & 294.2064 & $\mathrm{C}_{17} \mathrm{H}_{28} \mathrm{NO}_{3}{ }^{+}$ & 0.90 & nobilonine \\
\hline & & & & 249.1486 & 249.1485 & $\mathrm{C}_{15} \mathrm{H}_{21} \mathrm{O}_{3}^{+}$ & 0.38 & \\
\hline & & & & 221.1538 & 221.1536 & $\mathrm{C}_{14} \mathrm{H}_{21} \mathrm{O}_{2}{ }^{+}$ & 0.81 & \\
\hline & & & & 203.1434 & 203.1430 & $\mathrm{C}_{14} \mathrm{H}_{19} \mathrm{O}^{+}$ & 1.83 & \\
\hline & & & & 175.1480 & 175.1481 & $\mathrm{C}_{13} \mathrm{H}_{19}{ }^{+}$ & -0.57 & \\
\hline & & & & 161.0960 & 161.0961 & $\mathrm{C}_{11} \mathrm{H}_{13} \mathrm{O}^{+}$ & -0.62 & \\
\hline & & & & 133.1011 & 133.1012 & $\mathrm{C}_{10} \mathrm{H}_{13}{ }^{+}$ & -0.84 & \\
\hline & & & & 121.0643 & 121.0648 & $\mathrm{C}_{8} \mathrm{H}_{9} \mathrm{O}^{+}$ & -4.13 & \\
\hline & & & & 107.0498 & 107.0491 & $\mathrm{C}_{7} \mathrm{H}_{7} \mathrm{O}^{+}$ & 6.54 & \\
\hline 5 & 5.42 & 30 & 280.1909 & 280.1909 & 280.1907 & $\mathrm{C}_{16} \mathrm{H}_{26} \mathrm{NO}_{3}{ }^{+}$ & 0.53 & dendroamine \\
\hline & & & & 263.1876 & 263.1880 & $\mathrm{C}_{16} \mathrm{H}_{25} \mathrm{NO}_{2}{ }^{+}$. & -1.30 & \\
\hline & & & & 262.1801 & 262.1802 & $\mathrm{C}_{16} \mathrm{H}_{24} \mathrm{NO}_{2}{ }^{+}$ & -0.34 & \\
\hline & & & & 235.1927 & 235.1931 & $\mathrm{C}_{15} \mathrm{H}_{25} \mathrm{NO}^{+}$ & -1.53 & \\
\hline & & & & 220.1337 & 220.1332 & $\mathrm{C}_{13} \mathrm{H}_{18} \mathrm{NO}_{2}{ }^{+}$ & 2.03 & \\
\hline & & & & 207.1983 & 207.1982 & $\mathrm{C}_{14} \mathrm{H}_{25} \mathrm{~N}^{+}$ & 0.43 & \\
\hline & & & & 192.1387 & 192.1383 & $\mathrm{C}_{12} \mathrm{H}_{18} \mathrm{NO}^{+}$ & 1.98 & \\
\hline & & & & 178.1226 & 178.1226 & $\mathrm{C}_{11} \mathrm{H}_{16} \mathrm{NO}^{+}$ & 0.00 & \\
\hline & & & & 164.1435 & 164.1434 & $\mathrm{C}_{11} \mathrm{H}_{18} \mathrm{~N}^{+}$ & 0.34 & \\
\hline & & & & 136.1121 & 136.1121 & $\mathrm{C}_{9} \mathrm{H}_{14} \mathrm{~N}^{+}$ & 0.00 & \\
\hline 6 & 6.77 & 30 & 310.2024 & 310.2024 & 310.2013 & $\mathrm{C}_{17} \mathrm{H}_{28} \mathrm{NO}_{4}{ }^{+}$ & 4.01 & 6-hydroxynobi- \\
\hline & & & & 292.1931 & 292.1907 & $\mathrm{C}_{17} \mathrm{H}_{26} \mathrm{NO}_{3}^{+}$ & 9.08 & line \\
\hline & & & & 249.1484 & 249.1485 & $\mathrm{C}_{15} \mathrm{H}_{21} \mathrm{O}_{3}^{+}$ & -0.38 & \\
\hline & & & & 221.1536 & 221.1536 & $\mathrm{C}_{14} \mathrm{H}_{21} \mathrm{O}_{2}^{+}$ & 0.00 & \\
\hline & & & & 203.1428 & 203.1430 & $\mathrm{C}_{14} \mathrm{H}_{19} \mathrm{O}^{+}$ & -0.81 & \\
\hline & & & & 175.1483 & 175.1481 & $\mathrm{C}_{13} \mathrm{H}_{19}{ }^{+}$ & 0.81 & \\
\hline & & & & 161.0952 & 161.0961 & $\mathrm{C}_{11} \mathrm{H}_{13} \mathrm{O}^{+}$ & -4.11 & \\
\hline & & & & 133.1018 & 133.1012 & $\mathrm{C}_{10} \mathrm{H}_{13}{ }^{+}$ & 2.92 & \\
\hline & & & & 107.0482 & 107.0491 & $\mathrm{C}_{7} \mathrm{H}_{7} \mathrm{O}^{+}$ & -5.11 & \\
\hline 7 & 10.1 & 50 & 332.2593 & 332.2593 & 332.2584 & $\mathrm{C}_{21} \mathrm{H}_{34} \mathrm{NO}_{2}{ }^{+}$ & 2.71 & $N$-isopentenyl- \\
\hline & & & & 264.1965 & 264.1958 & $\mathrm{C}_{16} \mathrm{H}_{26} \mathrm{NO}_{2}^{+}$ & 2.65 & dendrobinium \\
\hline & & & & 246.1865 & 246.1852 & $\mathrm{C}_{16} \mathrm{H}_{24} \mathrm{NO}^{+}$ & 5.28 & \\
\hline & & & & 218.1900 & 218.1903 & $\mathrm{C}_{15} \mathrm{H}_{24} \mathrm{~N}^{+}$ & -1.37 & \\
\hline & & & & 176.1434 & 176.1434 & $\mathrm{C}_{12} \mathrm{H}_{18} \mathrm{~N}^{+}$ & 0.00 & \\
\hline & & & & 160.1116 & 160.1121 & $\mathrm{C}_{11} \mathrm{H}_{14} \mathrm{~N}^{+}$ & -3.12 & \\
\hline & & & & 119.0856 & 119.0855 & $\mathrm{C}_{9} \mathrm{H}_{11}{ }^{+}$ & 0.84 & \\
\hline & & & & 105.0709 & 105.0699 & $\mathrm{C}_{8} \mathrm{H}_{9}{ }^{+}$ & 9.52 & \\
\hline
\end{tabular}


Table 1 Positive ESI/QToF/MS/MS data of alkaloid compounds identified from D. nobile: $\mathbf{1}$ N-methyldendrobinium, 2 dendrobine, 3 mubironine B, 4 nobilonine, 5 dendramine, 6 6-hydroxynobiline, $7 \mathrm{~N}$-isopentenyldendrobinium, $8 \mathrm{~N}$-isopentenyl-6-hydroxydendroxinium, and $\mathbf{9} \mathrm{N}$-isopentenyldendroxinium. continued

\begin{tabular}{|c|c|c|c|c|c|c|c|c|}
\hline Peak & $\begin{array}{l}t_{R} \\
(\min )\end{array}$ & $\begin{array}{l}\text { CE } \\
\text { (ev) }\end{array}$ & $\begin{array}{l}\text { Precursor lon } \\
(\mathrm{m} / \mathrm{z})\end{array}$ & $\begin{array}{l}\text { Experimental } \\
\text { MS/MS ( } m / z)\end{array}$ & $\begin{array}{l}\text { Calculated } \\
\text { MS/MS (m/z) }\end{array}$ & $\begin{array}{l}\text { Elemental } \\
\text { composition }\end{array}$ & $\begin{array}{l}\text { Error } \\
\text { (ppm) }\end{array}$ & $\begin{array}{l}\text { Structural } \\
\text { elucidation }\end{array}$ \\
\hline \multirow[t]{8}{*}{8} & \multirow[t]{8}{*}{10.59} & \multirow[t]{8}{*}{50} & \multirow[t]{8}{*}{376.2482} & 376.2482 & 376.2482 & $\mathrm{C}_{22} \mathrm{H}_{34} \mathrm{NO}_{4}^{+}$ & 0.00 & \multirow{8}{*}{$\begin{array}{l}\mathrm{N} \text {-isopentenyl- } \\
\text { 6-hydroxyden- } \\
\text { droxinium }\end{array}$} \\
\hline & & & & 308.1850 & 308.1856 & $\mathrm{C}_{17} \mathrm{H}_{26} \mathrm{NO}_{4}^{+}$ & -1.95 & \\
\hline & & & & 290.1746 & 290.1751 & $\mathrm{C}_{17} \mathrm{H}_{24} \mathrm{NO}_{3}{ }^{+}$ & -1.72 & \\
\hline & & & & 275.1520 & 275.1516 & $\mathrm{C}_{16} \mathrm{H}_{21} \mathrm{NO}_{3}{ }^{+}$. & 1.45 & \\
\hline & & & & 262.1811 & 262.1802 & $\mathrm{C}_{16} \mathrm{H}_{24} \mathrm{NO}_{2}^{+}$ & 3.43 & \\
\hline & & & & 246.1120 & 246.1125 & $\mathrm{C}_{14} \mathrm{H}_{16} \mathrm{NO}_{3}{ }^{+}$ & -2.03 & \\
\hline & & & & 232.1329 & 232.1332 & $\mathrm{C}_{14} \mathrm{H}_{18} \mathrm{NO}_{2}{ }^{+}$ & -1.29 & \\
\hline & & & & 202.1229 & 202.1226 & $\mathrm{C}_{13} \mathrm{H}_{16} \mathrm{NO}^{+}$ & 1.48 & \\
\hline \multirow[t]{11}{*}{9} & \multirow[t]{11}{*}{11.42} & \multirow[t]{11}{*}{50} & \multirow[t]{11}{*}{360.2535} & 360.2535 & 360.2533 & $\mathrm{C}_{22} \mathrm{H}_{34} \mathrm{NO}_{3}{ }^{+}$ & 0.56 & \multirow{11}{*}{$\begin{array}{l}\mathrm{N} \text {-isopentenyl- } \\
\text { dendroxinium }\end{array}$} \\
\hline & & & & 292.1911 & 292.1907 & $\mathrm{C}_{17} \mathrm{H}_{26} \mathrm{NO}_{3}{ }^{+}$ & 1.37 & \\
\hline & & & & 274.1805 & 274.1802 & $\mathrm{C}_{17} \mathrm{H}_{24} \mathrm{NO}_{2}^{+}$ & 1.09 & \\
\hline & & & & 264.1958 & 264.1958 & $\mathrm{C}_{16} \mathrm{H}_{26} \mathrm{NO}_{2}^{+}$ & 0.00 & \\
\hline & & & & 262.1818 & 262.1802 & $\mathrm{C}_{16} \mathrm{H}_{24} \mathrm{NO}_{2}^{+}$ & 6.10 & \\
\hline & & & & 250.1442 & 250.1438 & $\mathrm{C}_{14} \mathrm{H}_{20} \mathrm{NO}_{3}{ }^{+}$ & 1.60 & \\
\hline & & & & 248.1997 & 248.2009 & $\mathrm{C}_{16} \mathrm{H}_{26} \mathrm{NO}^{+}$ & -4.83 & \\
\hline & & & & 246.1857 & 246.1852 & $\mathrm{C}_{16} \mathrm{H}_{24} \mathrm{NO}^{+}$ & 2.03 & \\
\hline & & & & 219.1247 & 219.1254 & $\mathrm{C}_{13} \mathrm{H}_{17} \mathrm{NO}_{2}{ }^{+}$ & -3.19 & \\
\hline & & & & 205.1454 & 205.1461 & $\mathrm{C}_{13} \mathrm{H}_{19} \mathrm{NO}^{+}$ & -3.41 & \\
\hline & & & & 176.1077 & 176.1070 & $\mathrm{C}_{11} \mathrm{H}_{14} \mathrm{NO}^{+}$ & 3.97 & \\
\hline
\end{tabular}

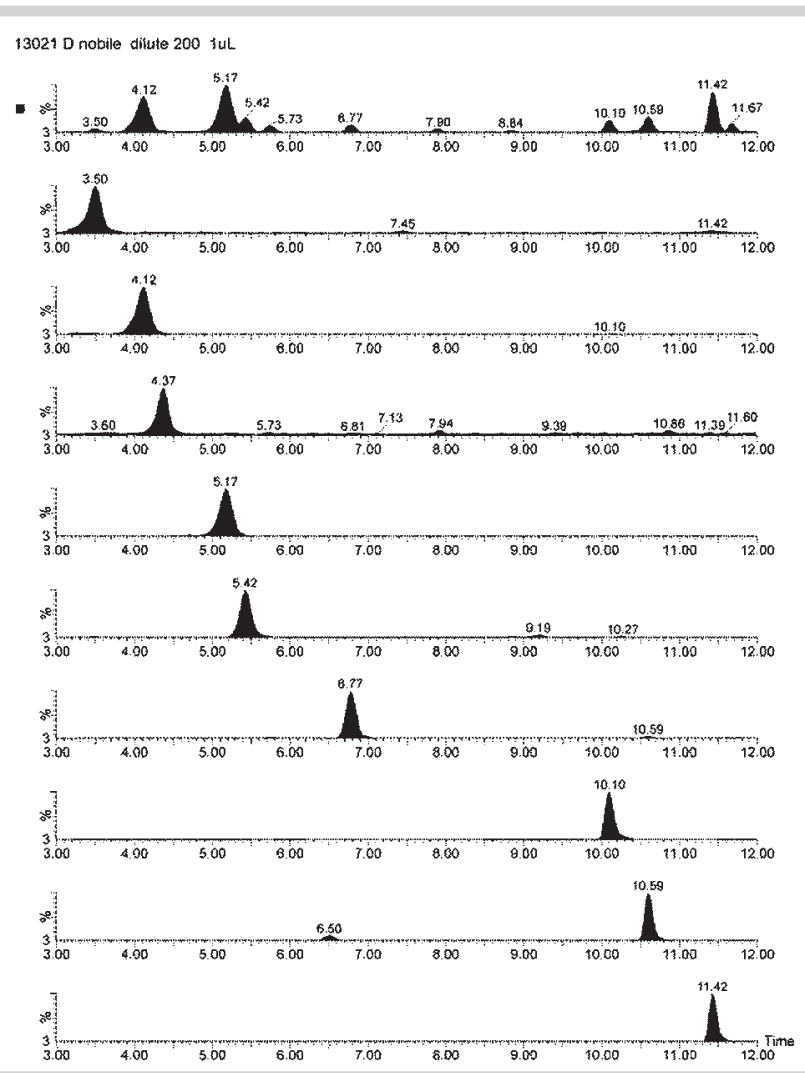

Fig. 2 Base peak ions (BPI) chromatogram of \#13021 D. nobile and extracted ion chromatograms (EIC) of individual sesquiterpene alkaloids: $1 \mathrm{~N}$-methyldendrobinium, 2 dendrobine, 3 mubironine B, 4 nobilonine, 5 dendramine, $\mathbf{6}$ 6-hydroxynobiline, $7 \mathrm{~N}$-isopentenyldendrobinium, $8 \mathrm{~N}$-isopentenyl-6-hydroxy-dendroxinium, and $\mathbf{9} \mathrm{N}$-isopentenyldendroxinium. from the whole plant of Dendrobium Snowflake "Red Star", a cultivar of D. nobile [15].

Compound 1 produced an $[\mathrm{M}]^{+}$ion at $\mathrm{m} / \mathrm{z} 278.2120\left(\mathrm{C}_{17} \mathrm{H}_{28} \mathrm{NO}_{2}{ }^{+}\right.$, calcd. 278.2115). Since the concentration of compound 1 was much lower than that of dendrobine $\mathbf{2}$ and compound $\mathbf{3}$, intensities of the fragment ions of compound $\mathbf{1}$ were not observed. On the basis of an elemental analysis, compound 1 was $14 \mathrm{Da}$ heavier than that of dendrobine $\mathbf{2}$, indicating an additional methylene moiety in 2. Overall, considering the identification of mubironine $B(3)$ and dendrobine $\mathbf{2}$ from this sample as well as a previous report from the literature [16], compound 1 could be tentatively identified as $\mathrm{N}$-methyldendrobinium.

Compound 5 produced ions with $\mathrm{m} / \mathrm{z} 280.1909\left([\mathrm{M}+\mathrm{H}]^{+}\right.$, $\mathrm{C}_{16} \mathrm{H}_{26} \mathrm{NO}_{3}{ }^{+}$, calcd. 280.1907), and the characteristic fragment ions at $\mathrm{m} / \mathrm{z} 262.1801,220.1337$, and 192.1387 were found in agreement with traits of the core skeleton of dendrobine-type alkaloids. The elemental composition showed that the $[\mathrm{M}+\mathrm{H}]^{+}$ion of compound $\mathbf{5}$ was $16 \mathrm{Da}$ heavier than that of dendrobine $\mathbf{2}$, which indicated that compound $\mathbf{5}$ was an oxygenated derivative of dendrobine. Therefore, compound $\mathbf{5}$ was tentatively identified as dendramine, a previously reported compound in D. nobile [17]. In the MS/MS spectra of compound $\mathbf{5}$, fragment ions at $\mathrm{m} / \mathrm{z}$ 263.1876, 235.1927, and 207.1983 could be radical ions corresponding to fragments $\mathrm{C}_{16} \mathrm{H}_{25} \mathrm{NO}_{2}{ }^{+\cdot}\left([\mathrm{M}+\mathrm{H}-\mathrm{OH}]^{+}\right), \mathrm{C}_{15} \mathrm{H}_{25} \mathrm{NO}^{+}$ $\left([263-\mathrm{CO}]^{+*}\right)$, and $\mathrm{C}_{14} \mathrm{H}_{25} \mathrm{~N}^{+\cdot}\left([235-\mathrm{CO}]^{+\cdot}\right)$, respectively. For dendrobine-type alkaloids, radical ions are likely formed when C-6 is oxidized.

A group of compounds $\left(t_{R}, 10.10,10.59\right.$, and $11.42 \mathrm{~min}$ ) had the common feature that a 68 Da fragment was dissociated from each compound, which corresponded to the loss of an isoamyl alkynyl moiety $\left(\mathrm{C}_{5} \mathrm{H}_{8}\right)$. Compound $7\left(t_{\mathrm{R}}, 10.10 \mathrm{~min}\right)$ was identified at $\mathrm{m} / \mathrm{z}$ 332.2593 ([M] $]^{+}, \mathrm{C}_{21} \mathrm{H}_{34} \mathrm{NO}_{2}{ }^{+}$, calcd. 332.2584). After application of a collision energy of $50 \mathrm{eV}$, a product ion at $\mathrm{m} / \mathrm{z} 264.1965$ $\left(\mathrm{C}_{16} \mathrm{H}_{26} \mathrm{NO}_{2}{ }^{+}\right)$corresponding to the loss of the $\mathrm{C}_{5} \mathrm{H}_{8}$ moiety from the molecular ion of compound $\mathbf{7}$ was generated. This product ion had the same elemental composition as the dendrobine $[\mathrm{M}+\mathrm{H}]^{+}$ ion. Interestingly, fragment ions at $\mathrm{m} / \mathrm{z} 264.1965,246.1865$, $218.1900,176.1434,160.1116,119.0856$, and 105.0709 were also 


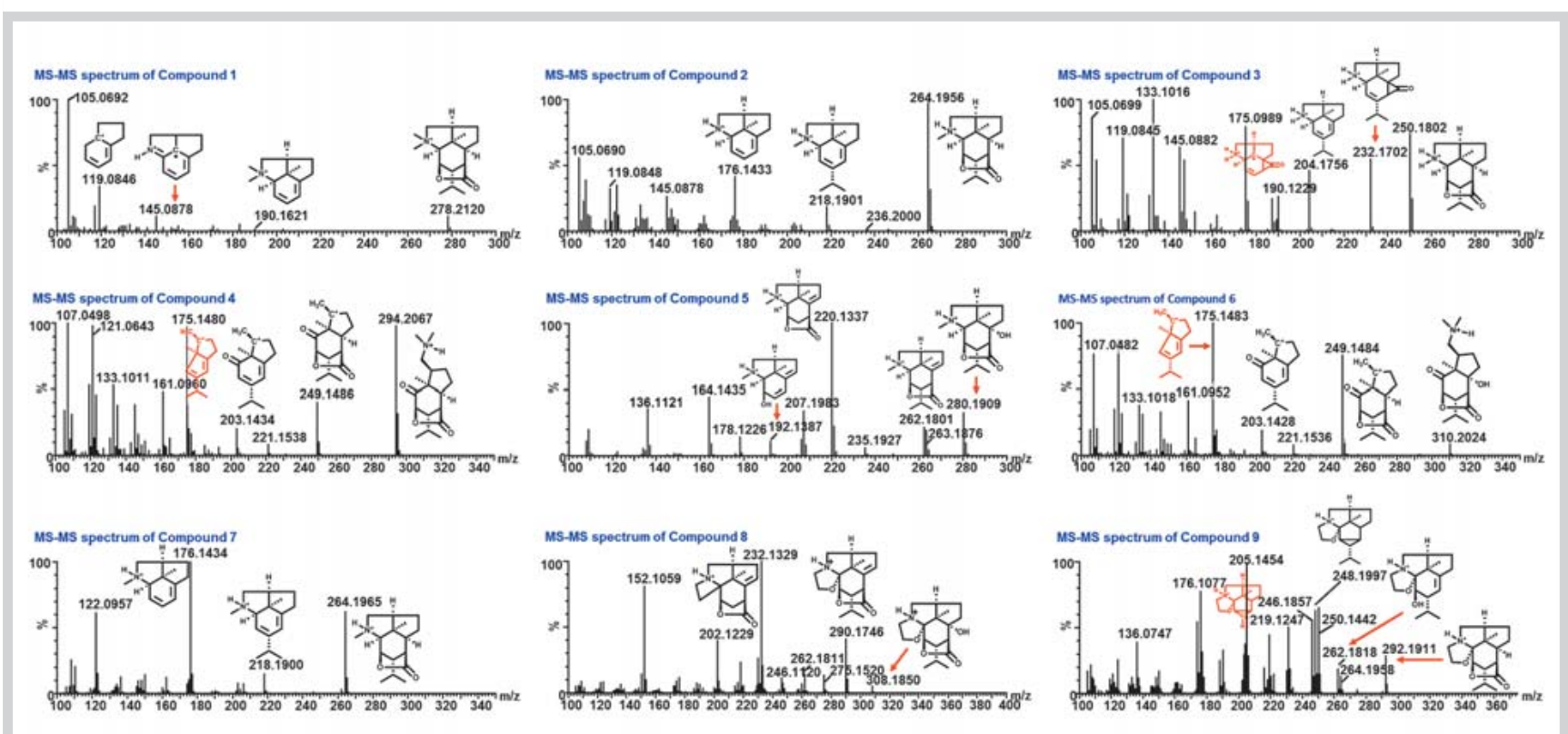

Fig. $3 \mathrm{MS} / \mathrm{MS}$ spectra of identified sesequiterpene alkaloids in $D$. nobile: $1 \mathrm{~N}$-methyldendrobinium, 2 dendrobine, 3 mubironine B, 4 nobilonine, 5 dendramine, 6 6-hydroxynobiline, $7 \mathrm{~N}$-isopentenyl-dendrobinium,
$8 \mathrm{~N}$-isopentenyl-6-hydroxy-dendroxinium, and $\mathbf{9} \mathrm{N}$-isopentenyldendroxinium (Color figure available online only).

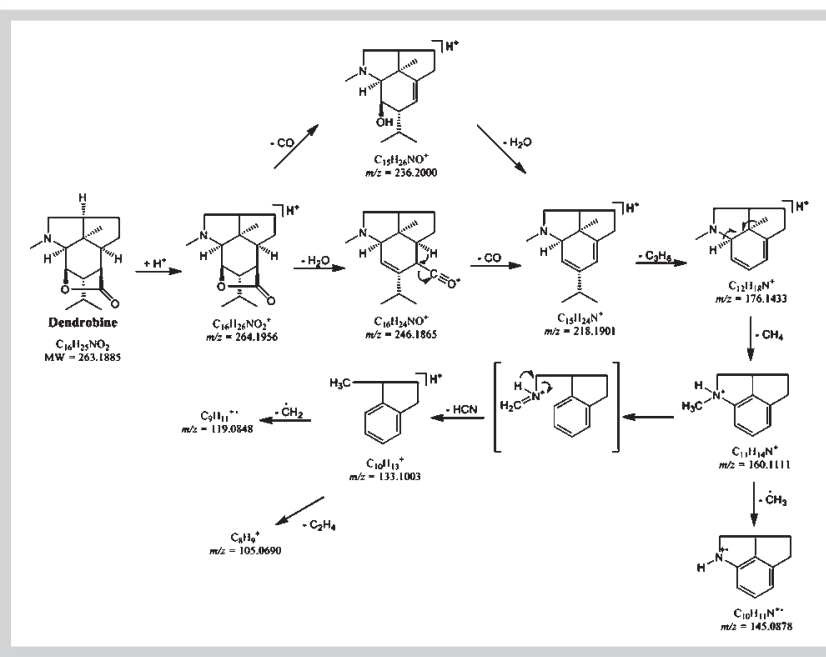

Fig. 4 Proposed fragmentation pathway of dendrobine (2).

identical to the fragment ions of dendrobine. Hence, compound 7 was tentatively characterized as $\mathrm{N}$-isopentenyldendrobinium [18].

Compound $9\left(t_{\mathrm{R},} 11.42 \mathrm{~min}\right)$ yielded ions at $m / z 360.2535\left([\mathrm{M}]^{+}\right.$, $\mathrm{C}_{22} \mathrm{H}_{34} \mathrm{NO}_{3}{ }^{+}$, calcd. 360.2533). The characteristic fragment ions at $m / z 292.1911,274.1805,264.1958$, and 250.1442 indicated that compound 9 had a core skeleton of dendrobine-type alkaloids. The product ion at $\mathrm{m} / \mathrm{z} 292.1911\left(\mathrm{C}_{17} \mathrm{H}_{26} \mathrm{NO}_{3}{ }^{+}\right)$corresponded to the loss of the $\mathrm{C}_{5} \mathrm{H}_{8}$ moiety from the molecular ion of compound 9. The elemental composition $\mathrm{C}_{17} \mathrm{H}_{26} \mathrm{NO}_{3}{ }^{+}$of the product ion at $\mathrm{m} / \mathrm{z} 292.1911$ was the same as the dendroxine $[\mathrm{M}+\mathrm{H}]^{+}$ion. Dendroxine has been previously reported in $D$. nobile [19]. Thus, compound 9 was tentatively characterized as

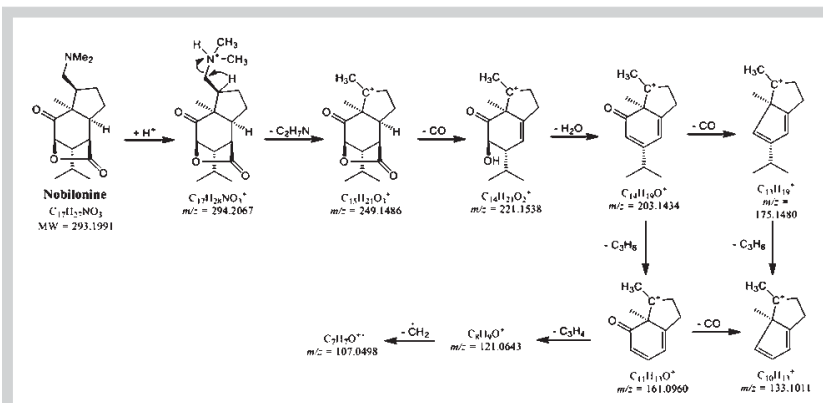

Fig. 5 Proposed fragmentation pathway of nobilonine (4).

$\mathrm{N}$-isopentenyldendroxinium, which was previously isolated from D. nobile, D. friedricksianum, and $D$. hildebrandii $[16,20]$.

Compound $8\left(t_{\mathrm{R}}, 10.59 \mathrm{~min}\right)$ produced ions at $\mathrm{m} / \mathrm{z} 376.2482\left([\mathrm{M}]^{+}\right.$, $\mathrm{C}_{22} \mathrm{H}_{34} \mathrm{NO}_{4}{ }^{+}$, calcd. 376.2482). The product ion at $\mathrm{m} / z 308.1850$ and fragment ions at $\mathrm{m} / \mathrm{z} 290.1746$ and 262.1811 indicated that compound 8 possessed an isopentenyl moiety and a core skeleton of dendrobine-type alkaloids. The elemental composition of compound 8 was 16 Da heavier than that of compound $\mathbf{9}$, indicating that compound $\mathbf{8}$ was an oxygenated derivative of compound $\mathbf{9}$. Furthermore, in the MS/MS spectra of compound 8, the peak at $\mathrm{m} / \mathrm{z} 275.1520$ was a radical ion corresponding to the loss of a $\mathrm{CH}_{3} \cdot$ moiety from the fragment ion at $\mathrm{m} / \mathrm{z} 290.1746$. Hence, compound 8 was tentatively identified as $N$-isopentenyl-6-hydroxydendroxinium, previously reported in D. friedricksianum, and D. hildebrandii [20].

Another subgroup of dendrobine alkaloids is nobilonine-type

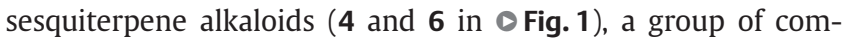
pounds derived from dendrobine. In this subgroup, the C2-C9 $\mathrm{N}$-heterocycle ring (A ring) is opened between $\mathrm{C} 2$ and $\mathrm{N}$, and a ketone is formed at C-2. 
Compound 4 was unambiguously identified as nobilonine from the methanolic extract of $D$. nobile from comparison with reference standards. The LOD of nobilonine was less than $30 \mathrm{ng} / \mathrm{mL}$. Nobilonine (compound 4 in $\bullet$ Fig. 2) was eluted from the column at $5.17 \mathrm{~min}$, and produced a protonated molecular ion at $\mathrm{m} / \mathrm{z}$ 294.2067 ([M + H $]^{+}, \mathrm{C}_{17} \mathrm{H}_{28} \mathrm{NO}_{3}{ }^{+}$, calcd. 294.2064). The CID of the $[\mathrm{M}+\mathrm{H}]^{+}$ions produced fragment ion $\mathrm{C}_{15} \mathrm{H}_{21} \mathrm{O}_{3}{ }^{+}$at $\mathrm{m} / z 249.1486$ after the neutral loss of the $\mathrm{C}_{2} \mathrm{H}_{7} \mathrm{~N}$ moiety from $\mathrm{C}-10$. Following that, the lactone ring was opened, and the $\mathrm{C}_{15} \mathrm{H}_{21} \mathrm{O}_{3}{ }^{+}$fragment successively yielded $\mathrm{C}_{14} \mathrm{H}_{21} \mathrm{O}_{2}{ }^{+}\left([249-\mathrm{CO}]^{+}\right)$and $\mathrm{C}_{14} \mathrm{H}_{19} \mathrm{O}^{+}([221-$ $\mathrm{H}_{2} \mathrm{O}^{+}$) at $\mathrm{m} / \mathrm{z} 221.1538$ and 203.1434, respectively. The fragment $\mathrm{C}_{14} \mathrm{H}_{19} \mathrm{O}^{+}$yielded key fragments at $m / z 175.1480\left(\mathrm{C}_{13} \mathrm{H}_{19}{ }^{+}\right)$ and $161.0960\left(\mathrm{C}_{11} \mathrm{H}_{13} \mathrm{O}^{+}\right)$, respectively, corresponding to the losses of moieties $\mathrm{CO}$ at $\mathrm{C}-2$ and $\mathrm{C}_{3} \mathrm{H}_{6}$ at $\mathrm{C}-3$. From ion 161.0960 $\left(\mathrm{C}_{11} \mathrm{H}_{13} \mathrm{O}^{+}\right)$, product ions corresponding to fragments $\mathrm{C}_{10} \mathrm{H}_{9} \mathrm{O}^{+}$ $\left(\left[161-\mathrm{CH}_{4}\right]^{+}\right), \mathrm{C}_{10} \mathrm{H}_{13}{ }^{+}\left([161-\mathrm{CO}]^{+}\right), \mathrm{C}_{8} \mathrm{H}_{9} \mathrm{O}^{+}\left(\left[161-\mathrm{C}_{3} \mathrm{H}_{4}\right]^{+}\right)$, and $\mathrm{C}_{7} \mathrm{H}_{7} \mathrm{O}^{+}\left(\left[121-\mathrm{CH}_{2}\right]^{+*}\right)$ were produced with $\mathrm{m} / \mathrm{z}$ 145.0651, $133.1011,121.0643$, and 107.0498, respectively. A proposed fragmentation pathway of nobilonine is illustrated in $\mathbf{~ F i g . ~} \mathbf{5}$. In the MS/MS spectra of nobilonine, the key fragments at $m / z 249,203$, 175,161 , and 121 are important for the characterization of nobilonine. The neutral losses of 18,28 , and 45 Da corresponding to the $\mathrm{H}_{2} \mathrm{O}, \mathrm{CO}$, and $\mathrm{C}_{2} \mathrm{H}_{7} \mathrm{~N}$ moieties, respectively, are characteristic for the determination of nobilonine-type alkaloids.

Compound 6 produced ions at $m / z 310.2024\left([\mathrm{M}+\mathrm{H}]^{+}, \mathrm{C}_{17} \mathrm{H}_{28} \mathrm{NO}_{4}{ }^{+}\right.$, calcd. 310.2013). Based on the analysis of elemental composition, compound 6 was 16 Da heavier than that of nobilonine 4 . This indicated that compound 6 was an oxygenated derivative of 4 . In the MS/MS spectra of compound $\mathbf{6}$, the product ion $(\mathrm{m} / \mathrm{z}$ 292.1931), corresponding to the loss of $\mathrm{H}_{2} \mathrm{O}$ from the protonated ion $[\mathrm{M}+\mathrm{H}]^{+}$, further proved that compound 6 contained a hydroxyl group. Moreover, characteristic fragment ions at $\mathrm{m} / \mathrm{z}$ 249.1484, 221.1636, 203.1428, 175.1483, and 161.0952 were found and indicated that compound 6 had a skeleton of nobilonine-type alkaloids. Based on the aforementioned information, compound 6 could be tentatively identified as 6-hydroxynobilonine, which has been previously isolated from $D$. hildebrandii and Dendrobium moniliforme (L.) Sw. [21,22].

Sample source and results for the identification and characterization of the nine sesquiterpene alkaloids from various samples are listed in Table 2 . A total of 26 samples including 19 plant samples and 7 dietary supplements were analyzed using the developed UHPLC-MS/MS method. As shown in $\odot$ Table 2, only sample \#13021 was an authentic plant sample, the rest of the 18 plant samples were commercial samples purchased either from local markets in China or from the Internet. Six of the D. nobile plant samples were analyzed. Among the five D. nobile commercial samples (\#8270, 10876, 10882, 10883, and 13034), two samples (\#8270 and \#10876) were found to contain eight of the nine alkaloids ( $N$-isopentenyl-6-hydroxydendroxinium 8 was not detected). The other thirteen Dendrobium plant samples did not contain any of these nine sesquiterpene alkaloids. Seven dietary supplements that were purchase from the Internet and claimed to contain either dendrobine alkaloids or $D$. nobile plant or both terms were tested. From these samples, none of dendrobine-type alkaloids were identified.

\section{Materials and Methods}

\section{$\nabla$}

\section{UHPLC chromatographic conditions}

The UHPLC analyses were performed on a Waters Acquity UPLC ${ }^{\mathrm{TM}}$ system including binary solvent manager, sampler manager, column compartment, and a PDA detector (Waters Acquity model code UPD). The separation was carried out on a Waters Acquity UPLC $^{\text {тм }}$ BEH Shield RP18 column $(100 \times 2.1 \mathrm{~mm}$ i.d., $1.7 \mu \mathrm{m})$ that was equipped with a guard column (Vanguard $2.1 \times 5 \mathrm{~mm}$ ). The sample temperature and column temperature were maintained at $15^{\circ} \mathrm{C}$ and $30^{\circ} \mathrm{C}$, respectively. The mobile phase consisted of water containing $0.05 \%$ formic acid (v/v) (A) and acetonitrile with $0.05 \%$ formic acid (B). The analysis was performed using the following gradient elution at a flow rate of $0.30 \mathrm{~mL} / \mathrm{min}$ : $0-8 \mathrm{~min}$, $5 \% \mathrm{~B}$ to $13 \% \mathrm{~B} ; 8-10 \mathrm{~min}, 13 \% \mathrm{~B}$ to $20 \% \mathrm{~B} ; 10-12 \mathrm{~min}, 20 \% \mathrm{~B}$ to $35 \% \mathrm{~B}$; and $12-18 \mathrm{~min}, 35 \% \mathrm{~B}$ to $100 \% \mathrm{~B}$. Each run was followed by a 4-min wash with $100 \% \mathrm{~B}$ and an equilibration period of 3.5 min with the initial conditions. Strong needle wash (90/10; acetonitrile/water, v/v) and weak needle wash solution (10/90; acetonitrile/water, $\mathrm{v} / \mathrm{v}$ ) were used. The total run time for analysis was $18 \mathrm{~min}$.

\section{ESI/ToF/MS/MS}

The high-resolution ESI-MS experiments were carried on a Waters ACQUITYTM XEVO QToF mass spectrometer that was connected to the UHPLC system via an ESI interface. The ESI source was operated in the positive ionization mode with the capillary voltage at $2.5 \mathrm{kV}$. The source and desolvation temperatures were set at 150 and $350^{\circ} \mathrm{C}$, respectively. The cone and desolvation gas flows were 50 and $900 \mathrm{~L} / \mathrm{h}$, respectively. The cone voltage was set at $35 \mathrm{~V}$. All data collected in the centroid mode were acquired using MassLynx ${ }^{\mathrm{TM}}$ NT 4.1 software. Mass accuracy of the parent and major fragments in this study was limited within 5 ppm, but few minor fragment ions were tolerated up to $10 \mathrm{ppm}$ when considering its limited peak intensity in the analysis. Leucine-enkephalin was used for the lock mass at a concentration of $2 \mathrm{ng} / \mathrm{mL}$ and flow rate of $5 \mu \mathrm{L} / \mathrm{min}$. Ions $[\mathrm{M}+\mathrm{H}]^{+}(\mathrm{m} / z$ 556.2771 Da $)$ and a fragment ion $(\mathrm{m} / z$ 278.1141 Da) of leucine-enkephalin were employed to ensure mass accuracy during the MS analysis. The lock spray interval was set at $30 \mathrm{~s}$, and the data were averaged over three scans. The mass spectrometer was programmed to step between low $(20 \mathrm{eV})$ and elevated (30-50 eV) collision energies on the gas cell, using a scan time of $0.1 \mathrm{~s}$ per function over a mass range of $50-900 \mathrm{~m} / \mathrm{z}$. When data were acquired with $\mathrm{MS}^{\mathrm{E}}$, two interleaved scan functions were used. The first scan function acquired a wide mass range using low collision energy. This scan function collected precursor ion information for the sample. The second scan function acquired data over the same mass range; however, the collision energy was ramped from low to high. This scan function allowed for the collection of a full-scan accurate mass fragments with precursor ion information. The mass spectrometer under the MS/MS tandem mode was programmed to use a scan time of $1 \mathrm{~s}$ per function scanned $50-500 \mathrm{~m} / \mathrm{z}$ and the collision energy applied to every precursor ( Table 1 ) was optimized for the best profile of the product ions. $\mathrm{MS}^{\mathrm{E}}$ and MS/MS data independent analysis provide accurate mass measurements of all detectable precursor and product ions, which are achieved by post-acquisition and lock mass corrections. 


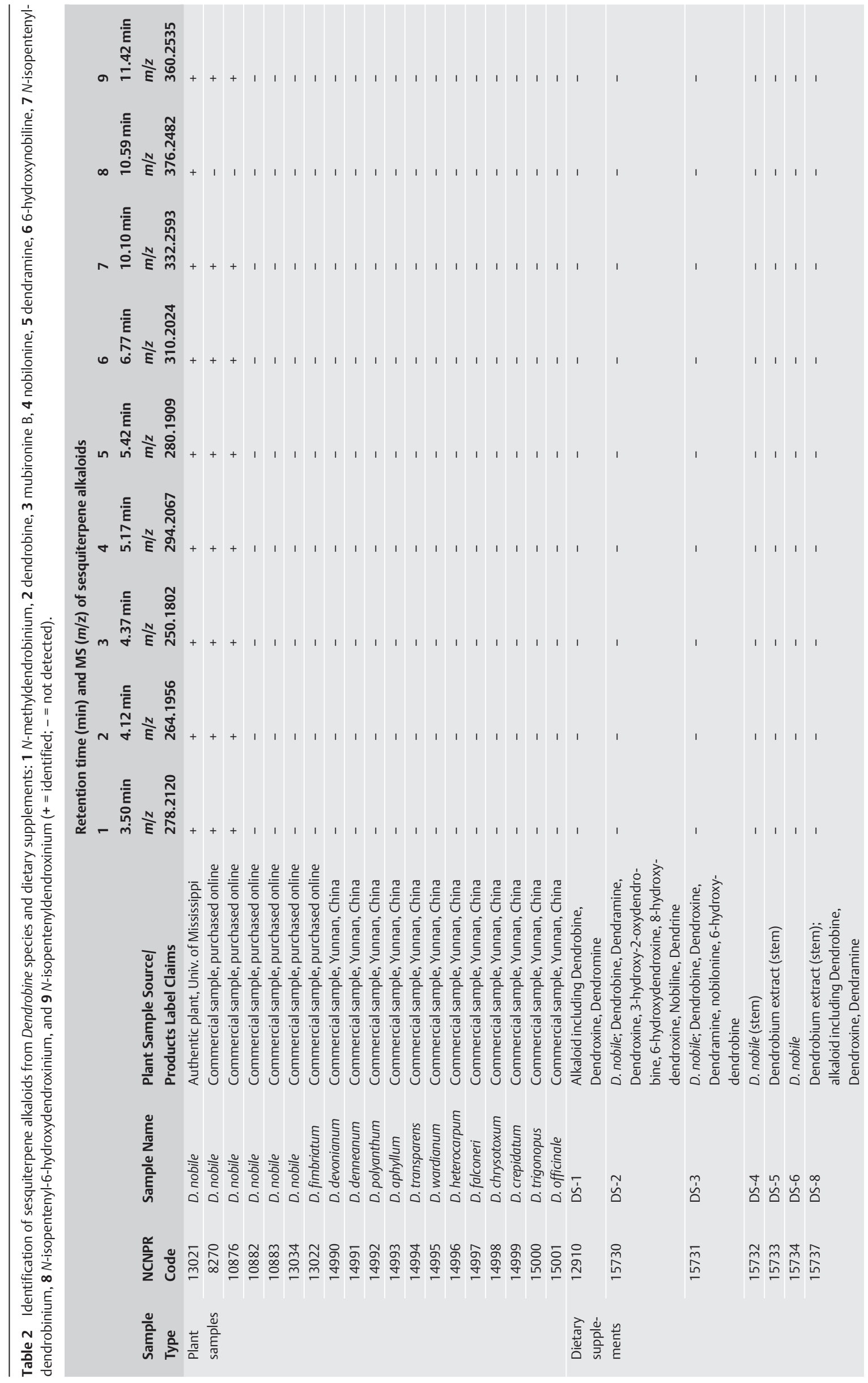




\section{Chemicals and reagents}

The reference compound dendrobine (2) was purchased from Quality Phytochemicals LLC with a purity greater than $98 \%$. Standard compound nobilonine (4) was isolated at the National Center for Natural Products Research (NCNPR), The University of Mississippi, University, MS, USA. The identity of nobilonine was determined by analysis of the spectral data $\left({ }^{1} \mathrm{H}\right.$ - and ${ }^{13} \mathrm{C}-\mathrm{NMR}$ and HR-ESIMS) [23], and the purity of nobilonine was confirmed to be above $95 \%$ by chromatographic methods.

HPLC grade methanol, acetonitrile, and formic acid were purchased from Fisher. Water for the HPLC mobile phase was purified using a Milipore Synergy Water Purification System.

\section{Plant materials}

Stems of D. nobile (NCNPR \#13021) were obtained from the cultivated, living collection of the Maynard W. Quimby Medicinal Plant Garden, The University of Mississippi. The plant material was identified by Dr. Aruna Weerasooriya of the Maynard W. Quimby Medicinal Plant Garden, The University of Mississippi, University, Mississippi, USA. Stems of Dendrobium devonianum (\#14990), Dendrobium denneanum Kerr (\#14991), Dendrobium polyanthum Wall. ex Lindl. (\#14992), Dendrobium aphyllum (Roxb.) C.E.C.Fisch. (\#14993), Dendrobium transparens Wall. ex Lindl. (\#14994), Dendrobium wardianum R.Warner (\#14995), Dendrobium heterocarpum Wall. ex Lindl. (\#14996), Dendrobium falconeri Hook. (\#14997), Dendrobium chrysotoxum Lindl. (\#14998), Dendrobium crepidatum Lindl. \& Paxton (\#14999), Dendrobium trigonopus Rchb.f. (\#15000), and Dendrobium officinale Kimura \& Migo (\#15001) were purchased from local markets in Yunnan Province, People's Republic of China and identified by Dr. Feng Wei of the Institute for Control of Traditional Chinese Medicine and Ethnic Medicine, National Institutes for Food and Drug Control, Beijing, China. Five plant samples (stems) of D. nobile (\#8270, 10876, 10882, 10883, and 13034), Dendrobium fimbriatum Hook. (\#13022), and seven dietary supplements (\#12910, \#15730-15734, and \#15737) claiming to contain D. nobile or dendrobine-type alkaloids were purchased online. Specimens of all samples are deposited at the Repository of Botanicals, NCNPR, The University of Mississippi, University, Mississippi, USA.

\section{Sample preparation}

Dry plant material was ground as fine powder. Five to ten tablets or capsules were weighed. The tablets (or capsule contents) were combined and ground with a mortar and pestle.

Dry powder of the plant samples $(0.5 \mathrm{~g})$ or an adequate amount of capsule/tablet/powder/pill contents were weighed and sonicated in $2.5 \mathrm{~mL}$ of $70 \%$ methanol (methanol: water $=70: 30, \mathrm{v} / \mathrm{v}$ ) for $30 \mathrm{~min}$, followed by centrifugation at $959 \times \mathrm{g}$ for $15 \mathrm{~min}$. The supernatant was transferred to a $10-\mathrm{mL}$ volumetric flask. The procedure was repeated three more times with $2.5 \mathrm{~mL} 70 \%$ methanol and the respective supernatants were combined. The final volume was adjusted to $10 \mathrm{~mL}$ with $70 \%$ methanol and mixed thoroughly. Prior to injection, an adequate volume of sample was passed through a $0.45-\mu \mathrm{m}$ PTFE filter and collected in an LC sample vial. For plant samples where a few constituents were highly concentrated, the sample solutions were further diluted up to 200 times.

\section{Acknowledgements}

$\nabla$

This research is supported in part by "Science Based Authentication of Dietary Supplements" funded by the Food and Drug Administration grant number 1U01FD004246-003, the United States Department of Agriculture, Agricultural Research Service, Specific Cooperative Agreement No. 58-6408-02-1-162-04.

\section{Conflict of Interest \\ $\nabla$}

The authors declare no conflict of interest.

\section{Affiliations}

${ }^{1}$ National Center for Natural Products Research, Research Institute of Pharmaceutical Sciences, School of Pharmacy, The University of Mississippi, University, MS, USA

2 Institute for Control of Traditional Chinese Medicine and Ethnic Medicine, National Institutes for Food and Drug Control, Beijing, People's Republic of China

${ }^{3}$ EISohly Laboratories, Incorporated, Oxford, MS, USA

${ }^{4}$ Division of Pharmacognosy, Department of BioMolecular Sciences,

School of Pharmacy, The University of Mississippi, University, MS, USA

${ }^{5}$ Department of Pharmacognosy, College of Pharmacy, King Saud University,

Riyadh, Saudi Arabia

\section{References}

1 Ye $\mathrm{Q}$ Qin G, Zhao W. Immunomodulatory sesquiterpene glycosides from Dendrobium nobile. Phytochemistry 2002; 61: 885-890

2 Chen X, Guo S. Advances in research of constituents and pharmacology of Dendrobium. Tianran Chanwu Yanjiu Yu Kaifa 2001; 13: 70-75

$3 \mathrm{Ng}$ TB, Liu J, Wong JH, Ye X, Wing SSC, Tong Y, Zhang KY. Review of research on Dendrobium, a prized folk medicine. Appl Microbiol Biotechnol 2012; 93: 1795-1803

4 Xu J, Han QB, Li SL, Chen XJ, Wang XN, Zhao ZZ, Chen HB. Chemistry, bioactivity and quality control of Dendrobium, a commonly used tonic herb in traditional Chinese medicine. Phytochem Rev 2013; 12: 341367

5 US Food and Drug Administration. Inspections, compliance, enforcement, and criminal investigations. Available at http://www.fda.gov/ ICECI/EnforcementActions/WarningLetters/ucm392485.htm. Accessed November 3, 2015

6 Yang $L$, Wang Z, Xu L. Simultaneous determination of phenols (bibenzyl, phenanthrene, and fluorenone) in Dendrobium species by high-performance liquid chromatography with diode array detection. J Chromatogr A 2006; 1104: 230-237

7 Zhou J, Xu Z, Kong H, Lu X, Xu G. Comparison of phenolic components among different species of Dendrobium (Shihu Fengdou) and determination of their active components, moscatilin and gigantol. Se Pu 2010; 28: 566-571

8 Xu B, Yang L, Wang Z. Quality evaluation of Dendrobii Caulis by HPLC. Zhongguo Yaoke Daxue Xuebao 2010; 41: 467-470

9 Li S, Wang C, Guo S. Determination of dendrobin in Dendrobium nobile by HPLC analysis. Zhongguo Yaoxue Zazhi 2009; 44: 252-254

10 Xu J, Zhao WM, Qian ZM, Guan J, Li SP. Fast determination of five components of coumarin, alkaloids and bibenzyls in Dendrobium spp. using pressurized liquid extraction and ultra-performance liquid chromatography. J Sep Sci 2010; 33: 1580-1586

11 Yang $L$, Wang $Y$, Zhang G, Zhang F, Zhang Z, Wang Z, Xu L. Simultaneous quantitative and qualitative analysis of bioactive phenols in Dendrobium aurantiacum var. denneanum by high-performance liquid chromatography coupled with mass spectrometry and diode array detection. Biomed Chromatogr 2007; 21: 687-694

12 Zhang G, Zhang F, Yang L, Zhu E, Wang Z, Xu L, Hu Z. Simultaneous analysis of trans- and cis-isomers of 2-glucosyloxycinnamic acids and coumarin derivatives in Dendrobium thyrsiflorum by high-performance liquid chromatography (HPLC)-photodiode array detection (DAD)electrospray ionization (ESI)-tandem mass spectrometry (MS). Anal Chim Acta 2006; 571: 17-24

13 Zha XQ Luo JP, Wei P. Identification and classification of Dendrobium candidum species by fingerprint technology with capillary electrophoresis. S Afr J Bot 2009; 75: 276-282 
14 Wang L, Wang C, Pan Z, Sun Y, Zhu X. Application of pyrolysis-gas chromatography and hierarchical cluster analysis to the discrimination of the Chinese traditional medicine Dendrobium candidum Wall. ex Lindl. J Anal Appl Pyrolysis 2011; 90: 13-17

15 Morita H, Fujiwara M, Yoshida N, Kobayashi J. New picrotoxinin-type and dendrobine-type sesquiterpenoids from Dendrobium Snowflake 'Red Star'. Tetrahedron 2000; 56: 5801-5805

16 Hedman K, Leander K. Studies on orchidaceae alkaloids. XXVII. Quaternary salts of the dendrobine type from Dendrobium nobile Lindl. Acta Chem Scand 1972; 26: 3177-3180

17 Okamoto T, Natsume M, Onaka T, Uchimaru F, Shimizu M. The structure of dendramine (6-hydroxydendrobine) and 6-hydroxydendroxine, the fourth and fifth alkaloid from Dendrobium nobile. Chem Pharm Bull (Tokyo) 1966; 14: 676-680

18 Leander K. Dendrobium (Orchidaceae) alkaloids. Chem Commun, Univ Stockholm 1973; 10: 31
19 Okamoto T, Natsume M, Onaka T, Uchimaru F, Shimizu M. The structure of dendroxine, the third alkaloid from Dendrobium nobile. Chem Pharm Bull (Tokyo) 1966; 14: 672-675

20 Hedman K, Leander K, Lüning B. Studies on orchidaceae alkaloids. XXV. $\mathrm{N}$-isopentenyl derivatives of dendroxine and 6-hydroxydendroxine from Dendrobium friedricksianum Lindl. and Dendrobium hildebrandii Rolfe. Acta Chem Scand 1971; 25: 1142-1144

21 Liu WH, Hua YF, Zhan ZJ. Moniline, a new alkaloid from Dendrobium moniliforme. J Chem Res 2007; 6: 317-318

22 Elander M, Leander K. Studies on orchidaceae alkaloids. XXI. 6-hydroxynobiline, a new alkaloid from Dendrobium hildebrandii Rolfe. Acta Chem Scand 1971; 25: 717-720

23 Okamoto T, Natsume M, Onaka T, Uchimaru F, Shimizu M. Further studies on the alkaloidal constituents of Dendrobium nobile (Orchidaceae). Structure determination of 4-hydroxy-dendroxine and nobilomethylene. Chem Pharm Bull 1972; 20: 418-421 